\title{
A Diferenciação dos Grupos 2 e 5 de Municípios Paulistas, Segundo o IPRS, a Partir das Transferências Constitucionais E DAS ReCEITAS TRIBUTÁRIAS: UMA APLICAÇÃO DA ANÁLISE DISCRIMINANTE
}

\author{
Milton Carlos Faria \\ Mestrado em Administração pela Escola de Administração de \\ Empresas de São Paulo Fundação Getulio Vargas - FGV. \\ milton_faria@uol.com.br
}

\author{
Maria Aparecida Gouvêa \\ Doutorado em Administração pela Faculdade de Economia, \\ Administração e Contabilidade da Universidade de São Paulo - USP \\ magouvea@usp.br
}

Patrícia Siqueira Varela

Mestrado em Controladoria e Contabilidade pela Faculdade de Economia, Administração e Contabilidade da Universidade de São Paulo - USP

psvarela@usp.br

\section{RESUmo}

Este estudo objetivou indicar se os municípios paulistas, especificamente os grupos de municípios caracterizados pelo Índice de Responsabilidade Social Paulista (IPRS) como municípios com alto índice de riqueza e baixos índices sociais (grupo 2) e municípios desfavorecidos em ambas as condições (grupo 5) podem ser diferenciados pelos valores per capita de transferência do Fundo de Participação dos Municípios (FPM), quota-parte do Imposto sobre Circulação de Mercadorias e Serviços (ICMS) e receita tributária arrecadada. A premissa foi a influência da distribuição do FPM no desempenho desses grupos ao servir como dispositivo de redistribuição de renda, compensando a menor capacidade tributária dos municípios desfavorecidos em riqueza. A avaliação dessas variáveis é uma forma de se analisar o desempenho dos municípios na área social em condições distintas economicamente e, para tanto, utilizou-se a técnica de análise discriminante. Os resultados evidenciam que os critérios de distribuição do FPM não contribuem de forma efetiva para a eqüidade das receitas disponíveis nesses grupos, ou então existem problemas de gestão na alocação dos recursos.

Palavras-chave: Análise Discriminante; Receitas Públicas; Índice Paulista de Responsabilidade Social; Longevidade; Escolaridade.
The object of this study is to indicate whether the municipalities of São Paulo State, specifically the groups of municipalities characterized by the State Social Responsibility Index as municipalities with high wealth index and low social indexes (group 2), and municipalities disfavored in both conditions (group 5) can be distinguished by per capita transference values from the Municipalities Participation Fund-MPF, Product and Service Circulation Tax Quota (PSCT) and collected tax revenue. The premise was the influence of the FPM distribution on the performance of those groups as it serves as a mechanism of income redistribution, conpensating for the lower tax capacity of the municipalities disfavored in wealth. The evaluation of these variables is one way to analyze the performance of the municipalities in the social area in economically distinct conditions, so to that end, the discriminant analysis technique was utilized. The results evince that the MPF distribution criteria do not contribute in an effective way to the available income fairness in both groups of municipalities studied, or there are management problems in the resources allocation.

Key words: Discriminant Analysis; tax revenues; São Paulo State Social Responsibility Index;Longevity; Education. 


\section{INTRODUÇÃO}

Uma das questões centrais da reforma do Estado, nas últimas décadas, é a radical modificação na regra relativa ao esquema de divisão social do trabalho, ou seja, na responsabilidade assumida pelos municípios e pelo setor privado, na produção de bens e serviços, antes considerada ocupação do Estado nacional (OSZLAK, 1998, p.53).

O movimento de descentralização tem sido uma estratégia utilizada tanto pelo processo de reforma do Estado quanto de redemocratização do país, favorecendo a transferência de poder, recursos e atribuições para os governos locais, mormente, quanto à implementação de políticas públicas.

Os municípios, depois da Constituição Federal de 1988, passaram a ter maior autonomia política e administrativa e, assumiram novos encargos na área social e na promoção do desenvolvimento econômico dentro da sua área de abrangência e, também, o desafio de estabelecer novas formas de relacionamento com a sociedade civil com o objetivo de aprofundar a democracia.

Os governos locais foram os principais beneficiários da descentralização fiscal, que foi iniciada na segunda metade dos anos setenta e reforçada pela Constituição de 1988, sobretudo com a ampliação das transferências federais e estaduais a esses entes da federação, o que incentivou a criação de vários municípios. O FPM - Fundo de Participação dos Municípios, federal, e a quota referente à parcela do repasse de ICMS - Imposto sobre a Circulação de Mercadorias e Serviços, estadual, são as principais transferências feitas aos municípios.

Para grande parte deles, essas duas transferências constitucionais representam a mais significativa fonte de financiamento das suas despesas. Bovo (2001, p. 114), afirma que para mais de 3.000 dos, aproximadamente, 5.550 municípios do país, 90\% dos seus recursos advém das transferências constitucionais, especialmente o FPM.

Ressalta-se, também, o fato de os principais tributos municipais - ISS (Imposto sobre Serviços) e IPTU (Imposto sobre a Propriedade Territorial Urbana) - apresentarem maior potencial de arrecadação nos médios e grandes municípios. Além disso, nos critérios de transferência da quota-parte de ICMS pertencente aos municípios (25\% do total arrecadado pelo estado), a intensidade da produção econômica exerce grande influência, ou seja, os valores transferidos estão relacionados com a capacidade de geração de riqueza no âmbito municipal. " [...] a lógica preponderante deste imposto é a de premiar os municípios economicamente mais bem-sucedidos." (ABRUCIO; COUTO, 1996, p.44).

Dessa forma, os critérios de distribuição dos recursos que compõem o FPM causam impactos significativos nas finanças dos municípios de pequeno porte. De acordo com o inciso II do art. 161 da Constituição Federal de 1988, cabe à lei complementar estabelecer regras sobre a entrega dos recursos do FPM, objetivando promover o equilíbrio sócio-econômico entre os municípios. O principal critério de rateio do FPM atualmente em vigor é o tamanho da população. Contudo, pode-se indagar se esse critério seria suficiente para promover o equilíbrio sócioeconômico, haja vista as diferenças entre os municípios não se restringirem, exclusivamente, a esse fator, mas também em termos da base econômica, nível de urbanização, condições físicas, capacidade de arrecadação de receitas tributárias, entre outros fatores, além da gestão adequada do recurso pelo município.

A análise da realidade dos governos locais do estado de São Paulo, sob a ótica do Índice Paulista de Responsabilidade Social (IPRS), indica a existência de grupos de municípios com combinações diferentes entre níveis de riqueza e indicadores de longevidade e escolaridade (FUNDAÇÃO SEADE, 2005a, p.10). Este trabalho faz parte de um conjunto de estudos que combina diferentes grupos e diferentes técnicas estatísticas para a análise dos dados. Neste caso, dois desses grupos chamam a atenção pelo fato de que os níveis diferentes de riqueza parecem não influir nos indicadores sociais, isto é, um dos grupos apresenta baixos níveis tanto na riqueza quanto nos indicadores sociais, enquanto que no outro grupo, com alto nível de riqueza, era de se esperarem bons indicadores sociais; porém, isso não ocorre. Uma das hipóteses levantadas é que a distribuição do FPM não apresenta influência na classificação dos diferentes grupos de municípios ao servir como dispositivo de redistribuição de renda.

Tomando-se por base a premissa de que os municípios com maior produção econômica e, conseqüentemente, com mais receitas tributárias recebem maiores transferências de ICMS, a transferência de FPM deve favorecer os municípios em condições mais adversas. Sendo assim, estabeleceu-se a seguinte questão orientadora do estudo:

As variáveis (i) receita tributária per capita, (ii) quota-parte de ICMS per capita e (iii) FPM per capita diferenciam grupos de municípios paulistas estabelecidos pelo IPRS?

O objetivo deste trabalho é verificar se alguns grupos de municípios paulistas, estabelecidos pelo IPRS, possuem médias diferentes de transferências de FPM, quota-parte de ICMS e receita tributária arrecadada, em termos per capita. Além disso, procura-se verificar a relação existente entre o conjunto dessas variáveis e a classificação dos municípios segundo o IPRS, por exemplo, a relação existente entre os municípios com baixo nível de riqueza e baixos índices de longevidade e escolaridade, quando comparados com os municípios que apresentam altos índices de riqueza e baixos índices de longevidade e escolaridade.

Espera-se contribuir para a discussão sobre a adequação dos critérios de distribuição do Fundo de Participação dos Municípios e para o aprofundamento do co- 
nhecimento da realidade dos municípios paulistas. Em particular, o intuito é indicar as variáveis de receitas públicas mais relevantes para discriminar os dois grupos de municípios focalizados.

\section{FUNDO DE PARTICIPAÇÃO DOS MUNICÍPI- OS - FPM}

A característica central da experiência brasileira quanto ao processo de descentralização foi a descoordenação, com conseqüências como o aumento das desigualdades socioeconômicas inter e intra-regional e inadequação da distribuição de encargos às três esferas federativas pela Constituição Federal de 1988, implicando a coexistência de lacunas ou superposição de funções (AFFONSO, 1996, p.9). Isso porque o processo de descentralização, iniciado no final dos anos setenta, foi comandado pelos estados e, principalmente, pelos municípios, no contexto de redemocratização, e não pelo governo federal (AFFONSO, 1996 , p.5).

Não obstante a indefinição da Constituição quanto à divisão de competências, estados e municípios acabaram assumindo novas responsabilidades por causa do maior volume de recursos disponíveis advindo da descentralização fiscal, pela diminuição dos gastos federais e pelas pressões da sociedade civil (AFFONSO, 1996, p.9).

Conforme Abrucio e Couto (1996, p.40), os municípios passaram a enfrentar um duplo desafio: assegurar condições mínimas de bem-estar social às suas populações (Função Welfare) e promover o desenvolvimento econômico com base em ações de âmbito local em parceria com a sociedade civil (Função Desenvolvimentista).

Para os autores, o enfrentamento dos desafios estaria condicionado por três parâmetros: a estrutura fiscal federativa, as diferenças socioeconômicas entre os municípios e a dinâmica política típica do âmbito municipal (ABRUCIO; COUTO, 1996, p.40).

O processo de descentralização fiscal, desencadeado nos anos setenta e com maior força no início dos anos oitenta, foi aprofundado pela Constituição Federal de 1988, tendo como principais conseqüências o aumento do poder de tributar das unidades subnacionais em sua própria jurisdição e o aumento de recursos disponíveis de forma não vinculada para os municípios, como resultado das transferências constitucionais, incluindo o Fundo de Participação dos Municípios e a participação na receita do ICMS (ABRUCIO; COUTO, 1996, p.42).

Apesar de os governos locais terem aumentado sua capacidade fiscal, tal processo não ocorreu de forma homogênea entre os diversos municípios brasileiros. Bovo (2001, p. 114) salienta o fato de as principais fontes de tributação dos municípios apoiarem-se no Imposto sobre Serviços - ISS, Imposto Predial e Territorial Urbano - IPTU e no Imposto sobre a Transmissão de Bens Imóveis - ITBI, tributos esses que apresentam maior potencial de arrecadação nos médios e grandes municípios, haja vista que nos de pequeno porte, com base eminentemente rural, a propriedade urbana é pouco significativa e o setor de serviços pouco expressivo.

"Um agravante a isto é a insuficiência dos mecanismos redistributivos existentes, sobretudo para o nível municipal" (ABRUCIO; COUTO, 1996, p.43). Os recursos transferidos pela União e pelos estados para os municípios deveriam servir como dispositivos para proporcionar condições eqüitativas aos municípios brasileiros para enfrentar os novos encargos sociais. Todavia, isso nem sempre acontece, como é o caso da quota-parte do ICMS que premia os municípios economicamente mais bem-sucedidos (ABRUCIO; COUTO, 1996, p.44).

Dessa forma, municípios mais ricos teriam maior capacidade de arrecadação de receitas e, conseqüentemente, maior capacidade de realizar investimentos nas áreas econômica e social. Vale ressaltar que a mudança no perfil econômico e social dos municípios não depende, exclusivamente, da obtenção de receitas públicas, mas de como tais recursos são aplicados e da complexidade dos problemas a serem enfrentados.

Sendo assim, a eficácia ou ineficácia redistributiva do Fundo de Participação dos Municípios exerce forte influência no desempenho municipal na área social. $O$ FPM é uma transferência constitucional feita pela União aos municípios, sendo este fundo constituído por $22,5 \%$ da arrecadação do Imposto de Renda - IR e do Imposto sobre Produtos Industrializados - IPI.

A transferência dos recursos que compõem o FPM divide-se em três partes:

- $10 \%$ do FPM total são distribuídos às capitais estaduais conforme coeficientes que consideram a população e o inverso da renda per capita do respectivo estado.

- 86,4\% do FPM total são distribuídos aos municípios do interior do país, de acordo com coeficientes definidos por faixa populacional no Decreto-Lei 1881/ 81.

- 3,6\% do FPM total são destinados à Reserva do Fundo de Participação dos Municípios que é distribuída entre os municípios do interior do país com coeficiente igual a 4,0 até o ano de 1998 e 3,8 a partir do exercício financeiro de 1999 . O recurso da reserva é um complemento aos valores recebidos conforme o item anterior e a distribuição ocorre de acordo com coeficientes que consideram a população e o inverso da renda per capita do respectivo estado.

Nos três casos, a participação de cada município é dada pela divisão do seu coeficiente pela soma dos coeficientes dos municípios brasileiros integrantes de cada grupo específico.

Segundo o $\int 4^{\circ}$ do Art. 91 do Decreto-Lei n ${ }^{\circ} 1.881 /$ 81 , os limites das faixas de número de habitantes serão reajustados sempre que, por meio de recenseamento 
demográfico geral, seja conhecida oficialmente a população total do país, estabelecendo-se o aumento percentual, com base no recenseamento imediatamente anterior.

De acordo com $\ 1^{\circ}$ do art. $1^{\circ}$ da Lei Complementar 91/97, ocorrerá a revisão das quotas de participação dos municípios anualmente com base nos dados oficiais de população produzidos pela Fundação Instituto Brasileiro de Geografia e Estatística - IBGE. Contudo, o $\$ 2^{\circ}$ do mesmo artigo, estabelece a manutenção dos coeficientes de participação do FPM de 1997 para os municípios que apresentarem redução dos seus coeficientes devido à perda de população decorrente das estimativas do IBGE. Os ganhos adicionais decorrentes de tal decisão estão sendo gradativamente eliminados desde 1999 e a previsão é de, em 2008, serem completamente eliminados.

Esquematicamente, os critérios de distribuição do FPM podem ser visualizados por meio da Figura 1, na qual coeficiente maior ou igual ao valor 3,8 indica municípios com população classificada nas duas maiores faixas populacionais:

Abrucio e Couto (1996, p.43) avaliam os critérios de distribuição do FPM como pouco eficientes, pois consideram o critério de renda apenas para as grandes cidades e as capitais estaduais.

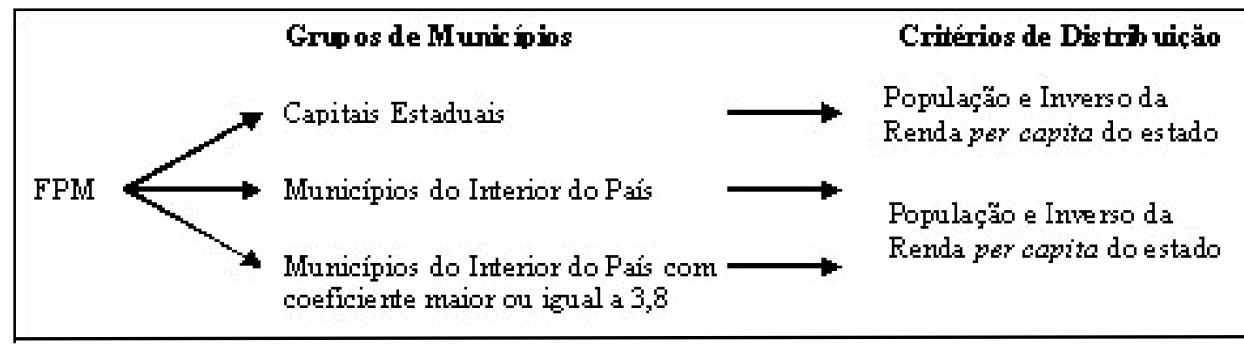

Figura 1: Critérios de Distribuição do FPM

Fonte: os autores

Nos demais municípios, o principal critério para a distribuição dos recursos componentes do FPM é o tamanho da população. Na verdade, os coeficientes de participação dos municípios são estabelecidos por faixas de população e não para um número específico, como visto na Tabela 1.

A amplitude das faixas e o fato de os coeficientes não aumentarem na mesma proporção do aumento das faixas de população ocasionam uma grande diferença entre os municípios se for considerado o FPM per capita, favorecendo os de menor porte.

Por exemplo, segundo dados do Sistema do Tesouro Nacional - STN (2005), 105 dos 645 municípios paulistas receberam transferência de FPM no valor de R\$ 1.908.104,63 em 2002. O menor município desse grupo (Borá) tinha uma população de 804 habitantes; portanto, recebeu FPM per capita anual de R $\$ 2.373,26$, enquanto o maior município do grupo (Estiva Gerbi), com população de 9.315 habitantes recebeu FPM per capita anual de
Tabela 1: Coeficientes Individuais de Participação do FPM

\begin{tabular}{lc}
\hline Faixa de Hab itautes (1960) & Coeficientes \\
\hline Até 10.168 & 0,6 \\
de 10.189 a 13.584 & 0,8 \\
de 13.585 a 16.980 & 1,0 \\
de 16.981 a 23.772 & 1,2 \\
de 23.773 a 30.564 & 1,4 \\
de 30.565 a 37.356 & 1,6 \\
de 37.357 a 44.148 & 1,8 \\
de 44.149 a 50.940 & 2,0 \\
de 50.941 a 61.128 & 2,2 \\
de 61.129 a 71.316 & 2,4 \\
de 71.317 a 81.504 & 2,6 \\
de 81.505 a 91.692 & 2,8 \\
de 91.623 a 101.680 & 3,0 \\
de 101.681 a 115.464 & 3,2 \\
de 115.465 a 129.048 & 3,4 \\
de 129.049 a 142.632 & 3,6 \\
de 142.632 a 156.216 & 3,8 \\
Alem de 156.216 & 4,0 \\
\hline
\end{tabular}

Fonte: Adaptado de Decreto-Lei n ${ }^{\circ} 1.881 / 81$, Art. $1^{\circ}$.

$\mathrm{R} \$ 204,84$. A diferença se deve ao critério de atribuição de um mesmo valor de FPM a municípios com tamanhos populacionais bem diferentes, mas pertencentes a uma mesma faixa populacional com grande amplitude de número de habitantes. Estas disparidades acontecem para todos os valores de receita de FPM referentes às diversas faixas e, portanto, agravam as diferenças entre os municípios e privilegiam os menores em detrimento dos demais.

Excluindo o município de São Paulo por ser a capital, Guarulhos recebeu o maior valor de FPM total, R\$ 17.871.002,58; em contrapar-tida, considerando-se a distribuição per capita, este município recebeu um dos menores valores, $\mathrm{R} \$ 15,78$, isso porque o montante de FPM não aumenta na mesma proporção do tamanho da população. Guarulhos é o segundo maior município do estado de São Paulo.

Num primeiro momento, pode parecer injusto o critério vigente para distribuição do FPM, visto que no exemplo de Borá e Estiva Gerbi, para uma mesma faixa de população, um município possui $\mathrm{R} \$ 2.373,26$ por habitante de receita de FPM e o outro apenas $\mathrm{R} \$ 204,84$. Estes não são casos isolados, existindo a tendência de os municípios maiores receberem menores valores per capita de FPM. Sendo assim, os municípios menos populosos teriam uma capacidade bem maior de investir na área social em relação aos mais populosos. Contudo, tal análise precisa ser feita com maior cuidado, pois existem diferenças quanto à capacidade fiscal dos municípios e quanto à 
gestão dos benefícios oriundos da distribuição da quota-parte de ICMS.

\section{3 ÍNDICE PAULISTA DE RESPONSABI- LIDADE SOCIAL}

No setor público, podem ser observadas diversas iniciativas e experiências de uso de indicadores sociais. A mais conhecida delas é a da Organização das Nações Unidas - ONU que, na década de noventa, criou o Índice de Desenvolvimento Humano - IDH, apresentando uma inovação ao introduzir, em sua concepção, as variáveis longevidade e educação, além da renda, para comparar o grau de desenvolvimento dos países.

Outras experiências surgiram a partir do IDH, como é o caso do Índice Paulista de Responsabilidade Social - IPRS. Tal índice foi construído pela Fundação Sistema Estadual de Análise de Dados (SEADE), órgão do governo do estado de São Paulo, em resposta à solicitação dos líderes e conselheiros do Fórum São Paulo - Século XXI para construção de índices que permitissem captar continuamente o progresso ou não do desenvolvimento dos municípios paulistas em direção à sociedade desejada e discutida amplamente no âmbito do Fórum.

O IPRS tem como objetivo a classificação dos municípios do estado de São Paulo segundo a qualidade de vida dos seus habitantes. Para isso, foram consideradas as três dimensões abrangidas pelo IDH (renda, longevidade e escolaridade), mas utilizando-se outras variáveis mais condizentes com a realidade municipal. A idéia inicial era usar indicadores que permitissem a avaliação não somente dos resultados e esforços empreendidos pelo poder público em prol do desenvolvimento em âmbito local, mas também a mensuração do grau de participação e controle da sociedade civil sobre tais ações.

Para a obtenção deste índice, os municípios do Estado de São Paulo foram classificados em grupos com características similares de riqueza, longevidade e escolaridade por meio da análise de agrupamentos (cluster analysis), grupos estes com as seguintes denominações: (1) municípios-pólo, (2) economicamente dinâmicos e de baixo desenvolvimento social, (3) saudáveis e de baixo desenvolvimento econômico, (4) de baixo desenvolvimento econômico e em transição social e (5) de baixo desenvolvimento econômico e social.

As variáveis consideradas em cada uma das dimensões do IPRS e a correspondente estrutura de ponderação utilizada estão sintetizadas na Tabela 2.
Tabela 2: Síntese das Variáveis Selecionadas e Estrutura de Pesos Adotada, segundo Dimensões do IPRS

\begin{tabular}{|c|c|c|}
\hline $\begin{array}{c}\text { Dimersia } \\
\text { do IPFs }\end{array}$ & Variáte is Seleciona das & $\begin{array}{l}\text { Contrib uiç:ĩo pan o } \\
\text { indica dor sin tétic o }\end{array}$ \\
\hline \multirow{6}{*}{$\begin{array}{l}\text { Fiqueza } \\
\text { Mutricipal }\end{array}$} & $\begin{array}{l}\text { Congumo tesidenoial de } \\
\text { enengia elétrioa }\end{array}$ & $44 \%$ \\
\hline & 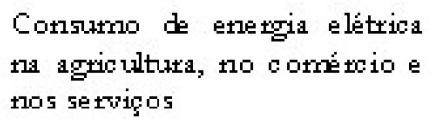 & $23 \%$ \\
\hline & 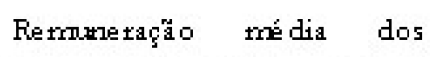 & $19 \%$ \\
\hline & $\begin{array}{l}\text { empregados oom oateira } \\
\text { assirnada do se tox publico }\end{array}$ & \\
\hline & $\begin{array}{l}\text { Walor adioionado fisoal per } \\
\text { os bida }\end{array}$ & $14 \%$ \\
\hline & Total petentatal & $100 \%$ \\
\hline \multirow{5}{*}{ Lomge vidade } & Mottalidade petinatal & $30 \%$ \\
\hline & Mottalidade itufantil & $30 \%$ \\
\hline & $\begin{array}{l}\text { Motalidade de pessoas de } 15 \\
\text { a } 39 \text { anos }\end{array}$ & $20 \%$ \\
\hline & $\begin{array}{l}\text { Moxtalidad de pessoas de } 60 \\
\text { anos e mais }\end{array}$ & $20 \%$ \\
\hline & Total petoetrtial & $100 \%$ \\
\hline \multirow{6}{*}{ Escolatidade } & 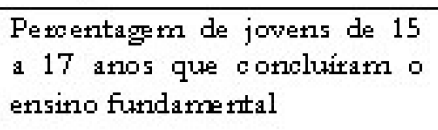 & $36 \%$ \\
\hline & $\begin{array}{l}\text { Pexoentagen de jowents de } 15 \\
\text { a } 17 \text { anos ootr1 pelo menos } \\
\text { quatto atros de eso olatidade }\end{array}$ & $8 \%$ \\
\hline & 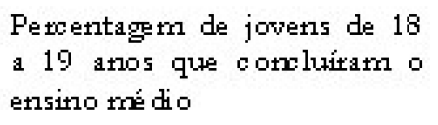 & $36 \%$ \\
\hline & $\begin{array}{l}\text { Penentagem de otiatpas de } \\
\text { dinco e seis anos que }\end{array}$ & $20 \%$ \\
\hline & freqüe ntamm pté-esc ola & \\
\hline & Total penetrtal & $100 \%$ \\
\hline
\end{tabular}

Fonte: Adaptado da Fundação SEADE, (2005b, p.5).

O indicador sintético de cada dimensão é o resultado da combinação das variáveis, sendo que o peso de cada uma delas na referida combinação foi obtido por meio de Análise Fatorial. Para facilitar o manuseio dos dados e a comparação de municípios, o indicador foi transformado em uma escala que varia de 0 a 100 .

Diferentemente do IDH em que o resultado final é um único indicador sintético, o IPRS utilizou os valores atribuídos a cada uma das dimensões (de 0 a 100) para aglomerar os municípios em grupos homogêneos internamente e heterogêneos entre si. A fundação SEADE sintetizou os indicadores de riqueza municipal, longevidade e escolaridade em escalas categóricas, as quais 
expressam o "padrão geral" dos grupos criados. A síntese dos critérios adotados para a formação dos grupos de municípios pelo IPRS está descrita no Quadro 1.

\begin{tabular}{|c|c|c|}
\hline $\begin{array}{l}\text { Grupos } \\
\text { do IPFS }\end{array}$ & $\begin{array}{l}\text { Criténos de Formaçáo dos } \\
\text { Grupos do IPRS }\end{array}$ & Descriçáno \\
\hline$G$ 土upo 1 & 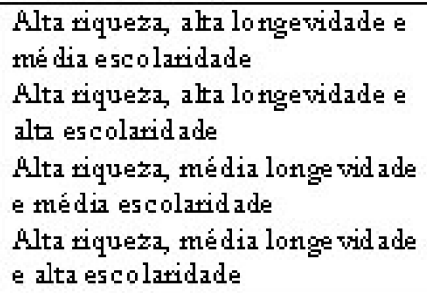 & 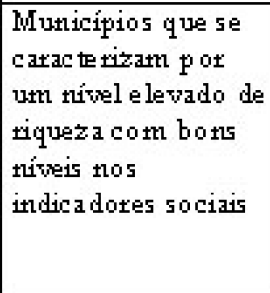 \\
\hline$G \pm 1+p$ o 2 & 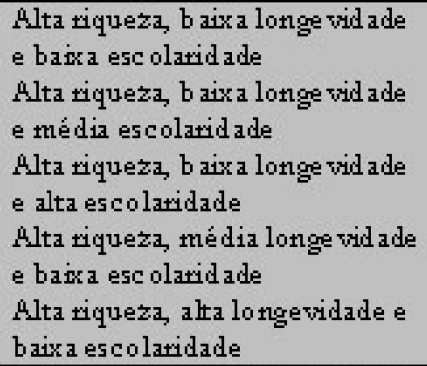 & 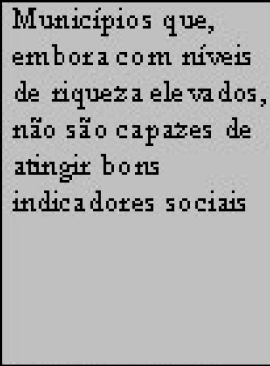 \\
\hline$G+1 p$ o 3 & 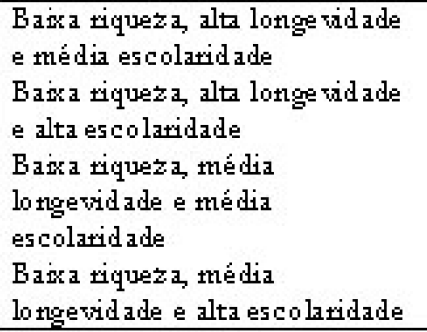 & 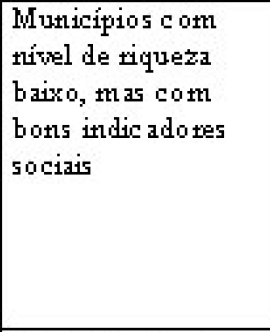 \\
\hline$G+1 p$ o 4 & 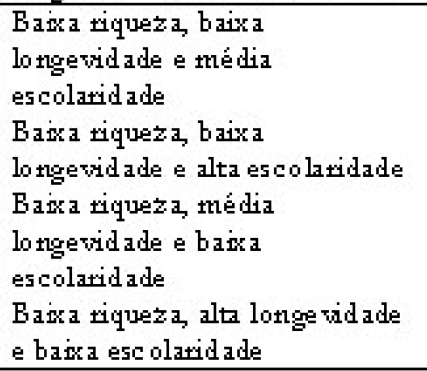 & 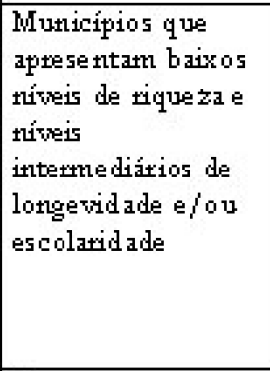 \\
\hline$G+7 p$ o 5 & $\begin{array}{l}\text { Baxa tiqueza, baira } \\
\text { longevidade e baix a } \\
\text { escolatidade }\end{array}$ & 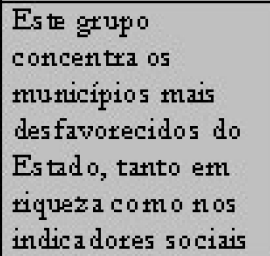 \\
\hline
\end{tabular}

Quadro 1: Critérios de Formação dos Grupos do IPRS

Fonte: Fundação SEADE, (2005b, p.10).

Pelo Quadro 1, é possível perceber as diferentes combinações entre níveis de riqueza municipal e de indicadores sociais. O grupo 2 chama a atenção por possuir alta riqueza municipal e, mesmo assim, não conseguir alcançar bons indicadores sociais, diferentemente, no contexto econômico, do grupo 5, o qual possui baixa riqueza.

O pior desempenho social dos dois grupos sinali- zado pelos indicadores de longevidade e escolaridade pode ser decorrente de diversas questões, como recursos insuficientes para atender à demanda da população, complexidade dos problemas sociais, desigualdades internas, problemas de gestão dos gastos públicos, entre outras.

Como já foi mencionado, os municípios com maior riqueza têm maior capacidade tributária; portanto, os dois grupos podem ter disponibilidade de recursos diferentes. Em outras palavras, os municípios do grupo 2 têm desempenho social parecido com o grupo 5, mas em uma situação mais desvantajosa, caso as transferências constitucionais não cumpram com seu papel redistributivo. Portanto, a indagação é se tais transferências, especialmente o FPM, não estão contribuindo para colocar os dois grupos em igualdade de condições de investimentos em políticas públicas.

\section{ASPECTOS METODOLÓGICOS}

Uma das formas de se analisar o desempenho dos municípios é avaliar a disponibilidade de recursos para a realização de investimentos sociais. Os recursos neste per capita de transferência do Fundo de Participação dos Municípios (FPM), quota-parte do Imposto sobre Circulação de Mercadorias e Serviços (ICMS) e receita tributária arrecadada. Os grupos foram formados de acordo com as notas atribuídas, aos municípios, para as dimensões do IPRS, que são: riqueza municipal, longevidade e escolaridade. Dessa forma, selecionaram-se alguns municípios e foi estabelecido um método de coleta e análise dos dados com características apresentadas nas seções a seguir.

\subsection{População}

A população objeto de estudo corresponde à capital e aos municípios do interior do estado de São Paulo pertencentes aos grupos específicos 2 e 5 do IPRS, que sinalizam a possibilidade de distribuição diferenciada do FPM, quota-parte de ICMS e receita tributária. Por esse motivo, surgiu o interesse na análise desses dois agrupamentos.

\subsection{Coleta de Dados}

Foram coletados dados referentes a quatro variáveis: FPM, Quota-parte de ICMS, Receita Tributária e Grupos de Municípios do IPRS.

Os dados do IPRS foram coletados no site da Fundação SEADE (2005b) e são referentes ao ano de 2002 e a todos os municípios do estado de São Paulo, ou seja, aos 645 municípios paulistas. Os dados das três fontes de receita pública, arrecadados no exercício de 2002 tiveram como fonte o site do Sistema do Tesouro Nacional (2005) e são relativos a 589 municípios paulistas. 


\subsection{Método de Análise dos Dados}

Com o intuito de aferir a significância da diferença dos valores per capita de tais receitas públicas nos dois grupos focalizados e identificar a variável com maior poder de discriminação entre estes grupos, aplicou-se a técnica estatística Análise Discriminante. Esta é a técnica apropriada para testar a hipótese de igualdade das médias de um conjunto de variáveis independentes ao longo de dois ou mais grupos (HAIR JR. et al., 2005, p. 209).

$\mathrm{Na}$ análise discriminante, as variáveis dependentes são categóricas e as variáveis independentes são intervalares ou razão e o objetivo principal é identificar o grupo a que um objeto, por exemplo, um município pertence. Sendo assim, a variável dependente é grupos2e5, que identifica o conjunto de municípios do grupo 2 e o do grupo 5 do IPRS, e as variáveis independentes são os valores per capita de FPM, Quota-parte de ICMS e Receita Tributária. Os valores per capita foram utilizados por refletirem a capacidade de investimento dos municípios em uma mesma dimensão, independentemente do tamanho da população.

Para a correta aplicação da técnica de análise discriminante, é preciso verificar o atendimento de algumas premissas inerentes à mesma. Tais suposições básicas podem ser resumidas em cinco itens: (1) ausência de outliers (observações atípicas), (2) normalidade das variáveis independentes, (3) linearidade das relações, (4) ausência de multicolinearidade entre as variáveis independentes e (5) igualdade nas matrizes de dispersão.

Antes de se verificarem todas estas condições, procedeu-se à análise de dados missing (dados perdidos), a ser comentada brevemente na seção 4.3.1.

\subsubsection{Tratamento dos Missing (Dados Perdidos)}

Em relação aos dados perdidos, o pesquisador deve se concentrar nos motivos que os geraram (HAIR JR. et al., 2005, p.56). No caso do Sistema do Tesouro Nacional, simplesmente não existiam informações para todos os 645 municípios paulistas, mas somente para 589. Segundo Hair Jr. et al. (2005, p.61), o tratamento mais simples e direto para lidar com dados perdidos é incluir no estudo somente as observações com dados completos e esta foi a decisão tomada neste trabalho.

\subsubsection{Tratamento dos Outliers (Observações Atípicas)}

O tratamento das observações atípicas foi feito sobre o total de 589 municípios, ao invés de se fazer para os municípios dos grupos 2 e 5 somente. A análise de todo o conjunto apresenta a vantagem de as variáveis independentes de cada município serem comparadas com as ob- servações de todos os municípios paulistas, uma vez que a classificação do IPRS abrange todo o estado.

A desvantagem de os 589 municípios serem levados em conta pode estar no fato de que se a análise fosse feita somente para os dados dos grupos 2 e 5 , poderiam ser identificadas menos observações atípicas. Todavia, seria um tratamento restrito dos dados e poderia gerar dúvidas quanto à sua legitimidade para a aplicação de técnicas multivariadas.

As observações atípicas podem ser classificadas em quatro classes (HAIR JR. et al., 2005, p.71):

a) observações atípicas por erro na entrada de dados ou falha na codificação;

b) observações devido a um evento extraordinário;

c) observações extraordinárias para as quais o pesquisador não tem explicação;

d) observações que estão no intervalo usual de valores para cada variável, porém são únicas em sua combinação de valores entre as variáveis.

Neste trabalho, cinco casos foram eliminados por apresentarem valores muito discrepantes de FPM total, indicando erros na entrada de dados.

Ocorreram também casos peculiares, como o da capital de São Paulo com valor muito baixo de FPM per capita e muito alto para Receita Tributária per capita. Para identificar estes casos, as variáveis independentes dos 584 municípios restantes foram transformadas mediante logaritmo natural (para se obter normalidade na distribuição de cada variável) e padronização de escala (segundo o método Z-scores) e, em seguida, eliminaram-se os casos fora do limite de mais ou menos dois desvios padrão em relação à média.

Os municípios dos grupos 2 e 5 incluídos na análise são os que permaneceram após o tratamento dos dados missing e outliers. Como resultado, o grupo 2 apresentou 55 municípios e o grupo 5, 84 municípios, com um total dos dois grupos de 139 municípios.

\subsubsection{Distribuição Normal}

A suposição mais importante em análise multivariada e em específico na análise discriminante é a suposição de normalidade das variáveis independentes envolvidas (HAIR JR. et al., 2005, p.220).

Foram utilizados testes estatísticos para avaliar a normalidade com base em assime-tria e curtose, cujos resultados encontram-se na Tabela 3 . Tais resultados foram confrontados, em módulo, com o valor de

Tabela 3: Teste de Assimetria e Curtose

\begin{tabular}{cccc}
\hline Teste & fpm & rect & icms \\
\hline Assimetria & 2,0562 & 1,1568 & 0,1733 \\
Curtose & 1,8536 & $-1,3295$ & $-1,7686$ \\
\hline
\end{tabular}

Fonte: Elaborado pelos autores. 
significância de 1,96. As variáveis foram consideradas simétricas, exceto a variável fpm. No teste de curtose as variáveis apresentaram forma mesocúrtica.

Foram feitos os testes de aderência à curva normal de Kolmogorov-Smirnov para as variáveis independentes, obtendo-se os seguintes níveis de significância: fpm 0,218; icms 0,540 e rect 0,806 , que são valores superiores ao nível de significância 0,05 pré-estabelecido e indicam não rejeição da hipótese de distribuição normal dos dados. Assim, confirma-se a aderência à curva normal das 3 variáveis, sendo que a menor qualidade de ajuste ocorreu para a variável fpm.

\subsubsection{Outros Pressupostos da Análise Discriminante}

As suposições de linearidade das relações, ausência de multicolinearidade entre as variáveis independentes e igualdade nas matrizes de dispersão foram verificadas no decorrer do processamento da análise discriminante. Por uma questão de simplificação, será considerado como premissa que as variáveis deste estudo guardam entre si uma relação de linearidade e, com esta propriedade atendida, será pertinente a aplicação da análise discriminante. A ausência de multicolinearidade foi tratada com o uso do método stepwise. A igualdade nas matrizes de dispersão foi avaliada pelos testes Levene (contexto univariado) e Box'M (contexto multivariado).

Por uma questão de simplificação, nos comentários das variáveis focalizadas neste estudo serão, em geral, usados os termos originais a elas associados (FPM per capita, Quota-parte de ICMS per capita e Receita Tributária per capita), porém, na verdade, trata-se dos seus valores submetidos ao logaritmo natural e padronização pelo método Z-scores.

\section{ANÁLISE DOS RESULTADOS}

A questão central inerente à análise discriminante é a seguinte: as variáveis FPM per capita, Quota-parte de ICMS per capita e Receita Tributária per capita discriminam os grupos 2 e 5 ? Ou seja, a média de cada uma dessas variáveis é diferente nestes dois grupos?

Nesta seção, primeiramente serão apresentadas algumas estatísticas univariadas e depois as estatísticas no contexto multivariado.

\subsection{Análise Univariada}

\subsubsection{Estatísticas Descritivas}

A Tabela 4 indica algumas estatísticas descritivas em cada grupo.
Tabela 4: Estatísticas Descritivas

\begin{tabular}{|c|c|c|c|}
\hline & Variaite is & Médin & Des vio Padrío \\
\hline \multirow[t]{3}{*}{ Grupo 2} & $\mathrm{f}_{\mathrm{f}+\mathrm{nI}}$ & $-0,5665$ & 0,5763 \\
\hline & is trus & $-0,0962$ & 0,8526 \\
\hline & teot & 0,6721 & 0,6902 \\
\hline \multirow[t]{3}{*}{ Grupo 5} & $\mathrm{f}_{\mathrm{F}+\mathrm{r} 1}$ & 0,1167 & 0,5282 \\
\hline & is $\mathrm{mrr}$ & $-0,4686$ & 0,8022 \\
\hline & teet & $-0,43,48$ & 0,7833 \\
\hline \multirow[t]{3}{*}{ Total } & $f_{\mathrm{P}+\mathrm{m} 1}$ & $-0,1536$ & 0,6405 \\
\hline & in $\mathrm{mrzs}$ & $-0,3213$ & 0,8396 \\
\hline & teot & 0,0032 & 0,9222 \\
\hline
\end{tabular}

Fonte: Elaborado pelos autores.

Nota-se que no grupo 2 a média das variáveis FPM per capita e Quota-parte de ICMS per capita são negativas e a média da variável Receita Tributária per capita é positiva. O oposto ocorre no grupo 5, exceto para a variável Quota-parte de ICMS per capita.

As médias negativas das variáveis FPM per capita e Quota-parte de ICMS per capita no grupo 2 indicam menor transferência de tais receitas para os municípios considerados mais ricos. A riqueza é comprovada pela média positiva da Receita Tributária per capita. Raciocínio oposto ocorre com a análise das médias do grupo 5 que é classificado como de baixa riqueza, isto é, apresenta média positiva de FPM per capita e médias negativas da Quota-parte de ICMS per capita e da Receita Tributária per capita.

As médias das variáveis fornecem indícios de distinção entre os grupos 2 e 5 . Observe-se que os desvios padrão apresentam valores muito altos, evidenciando grande heterogeneidade dentro de cada grupo. Para uma correta comparação das médias dos grupos para cada variável, realizou-se o teste de igualdade das médias apresentado a seguir.

\subsubsection{Teste de Igualdade das Variâncias e das Médias}

Para testar a hipótese de as variâncias de cada variável serem homogêneas nos dois grupos, utilizou-se o teste de Levene e para testar a hipótese de igualdade das médias de cada variável nos dois grupos, realizou-se o teste t de student. Pela Tabela 5, as variâncias podem ser consideradas iguais e as médias diferentes, para um nível de significância de 0,05 .

Tabela 5: Testes: Levene e t das Médias das Variáveis Transformadas

\begin{tabular}{lcc|ccc}
\hline & \multicolumn{2}{c|}{ Tes te Levere } & \multicolumn{3}{c}{ Teste t } \\
\hline & $\mathrm{F}$ & Sig. & $\mathrm{T}$ & $\mathrm{Df}$ & Sig. \\
F pm & 0,502 & 0,460 & $-7,202$ & 137 & 0,000 \\
Icms & 0,000 & 0,985 & 2,609 & 137 & 0,010 \\
Fect & 0,549 & 0,460 & 8,529 & 137 & 0,000 \\
\hline
\end{tabular}

Fonte: Elaborado pelos artores. 
Estes resultados, no contexto univariado, são favoráveis, de um modo geral, ao uso da análise discriminante. A expectativa na análise multivariada também será variâncias iguais nos grupos e médias diferentes.

\subsection{Análise Multivariada}

\subsubsection{Avaliação da Multicolinearidade}

A análise das correlações entre as variáveis explicativas está na Tabela 6 a seguir:

Tabela 6: Matriz de Correlação dos Grupos

\begin{tabular}{cccc}
\hline & fpm & icms & rect \\
$\mathbf{f p m}$ & 1,000 & 0,414 & $-0,325$ \\
icms & 0,414 & 1,000 & $-0,163$ \\
rect & $-0,325$ & $-0,163$ & 1,000 \\
\hline
\end{tabular}

Fonte: Elaborado pelos autores.

As correlações que podem ser consideradas altas e comprovadas por meio do teste de significância com nível crítico de 0,05, são: Quota-parte ICMS per Capita com FPM per capita $(0,414)$ e Receita Tributária per capita com FPM per capita (-0,325). A correlação entre Quota-parte de ICMS per capita e Receita Tributária per capita pode ser considerada baixa $(-0,163)$. A preocupação aqui é que não haja problemas de multicolinearidade. Os resultados mostram que os recursos provenientes da União (FPM) e do estado (Quota-parte de ICMS) apresentam correlação expressiva e positiva, isto é, municípios com maior recurso da União também têm maior recurso do estado e vice-versa. O recurso FPM se correlaciona negativamente com a Receita Tributária, isto é, maior recurso próprio do município significa menor recurso proveniente da União.

Com relação à suposição de que a Quota-parte de ICMS premia os municípios mais bem sucedidos economicamente, no caso os do grupo 2 (conforme Tabela 4), os dados per capita indicam o oposto, tal variável e a Receita Tributária per capita se correlacionam negativamente, apesar de o valor ser considerado baixo, porém significativo, de acordo com o teste de significância.

Foi empregado o método stepwise. Dessa maneira, se as correlações estivessem estatisticamente muito acentuadas em módulo, a ponto de apresentarem problemas de multicolinearidade, o processamento da análise discriminante somente incluiria as variáveis independentes com níveis toleráveis de correlação.

\subsubsection{Teste de Igualdade das Variâncias}

Conforme a Tabela 7, o teste Box'M apresentou significância 0,189 , isto é, não se rejeita $H_{0}$, para um nível de significância de 0,05 , o que indica que as matrizes de covariância dos dois grupos são semelhantes, ou seja, são provenientes de populações iguais nos dois grupos, o que atende a uma das premissas da técnica de análise discriminante.

Tabela 7: Resultados do Teste Box'M

\begin{tabular}{lc}
\hline Borm & 8,958 \\
F aptoxima do & 1,455 \\
Af1 & 6 \\
If2 & $895,56,024$ \\
Signific atro ia & 0,189 \\
\hline
\end{tabular}

Fonte: Elaborado pelos autores.

\subsubsection{Teste de Igualdade das Médias}

O resultado do teste da igualdade da média nos dois grupos de municípios, considerando-se cada variável independente e a estatística Wilks'Lambda, encontra-se na Tabela 8.

Tabela 8: Teste de Igualdade das Médias dos Grupos

\begin{tabular}{cccccc}
\hline & Wdrk' Lamb b & F & dfl & df & Significância \\
F pm & 0,726 & 51,724 & 1 & 137 & 0,000 \\
Icms & 0,953 & 6,813 & 1 & 137 & 0,010 \\
Rect & 0,653 & 72,792 & 1 & 137 & 0,000 \\
\hline
\end{tabular}

Fonte: Elaborado pelos autores.

O teste Wilks' Lambda indica que as médias das variáveis são consideradas diferentes nos grupos 2 e 5 , ao nível de significância de 0,05 . Observa-se ainda que a variável Receita Tributária per capita é a que tem diferenças mais acentuadas entre as médias dos grupos, pois apresenta o menor valor da estatística Wilk's Lambda. Esta é uma das razões para o fato de Receita tributária per capita ter sido a primeira variável incluída na seleção pelo método stepwise.

\subsubsection{Seleção das Variáveis}

Procedeu-se à aplicação da análise discriminante com o uso do método stepwise e o critério Wilk's Lambda, com probabilidades proporcionais ao tamanho dos grupos de municípios. Pelo método stepwise, as variáveis são incluídas, uma de cada vez, com base no seu poder discriminatório, conforme a Tabela 9: 
Tabela 9: Variáveis Incluídas pelo Método Stepwise segundo a Estatística Wilks'Lambda

\begin{tabular}{cccccccccc}
\hline Pass o & Inchida & Es tatí tica & dfl & De & df & Es tat exata F & dfl & df & Sig. \\
\hline $\mathbf{1}$ & Rect & 0,653 & 1 & 1 & 137 & 72,792 & 1 & 137 & 0,000 \\
2 & FPm & 0,591 & 2 & 1 & 137 & 46,970 & 2 & 136 & 0,000 \\
3 & Icms & 0,503 & 3 & 1 & 137 & 44,379 & 3 & 135 & 0,000 \\
\hline
\end{tabular}

Fonte: Elaborado pelos autores.

O método stepwise prioriza a inclusão de variáveis com alto poder discriminatório e que sejam menos correlacionadas entre si. A análise incluiu as três variáveis na seguinte ordem: primeiro a variável Receita Tributária per capita, em segundo lugar FPM per capita e em terceiro lugar a variável Quota-parte de ICMS per capita. O valor da estatística Wilks' Lambda, no primeiro passo, é igual ao da variável Receita Tributária per capita obtido na Tabela 8. Com a inclusão das demais variáveis, houve uma diminuição do valor dessa estatística, evidenciando uma melhoria na discriminação dos grupos com o uso conjunto de mais de uma variável explicativa. Chegou-se, por fim, ao valor 0,503 . A discriminação entre os grupos proporcionada pelas variáveis explicativas é estatisticamente significante, de acordo com o teste $\mathrm{F}$ aplicado nos resultados da estatística Wilks' Lambda em cada passo de inclusão de tais variáveis na função discriminante.

A Receita Tributária significa recurso próprio do município e, portanto, é um dado mais genuíno do que a Quota-parte de ICMS e FPM que são alocações do estado e da União, segundo critérios estabelecidos. Esse pode ter sido o fato de a Receita Tributária per capita ter sido escolhida em primeiro lugar na análise discriminante pelo método stepwise. A análise discriminante indica a necessidade de utilização das três variáveis para explicar a diferenciação dos grupos 2 e 5 de municípios e, dessa forma, a questão central que norteou o desenvolvimento deste estudo está respondida.

As cargas discriminantes, apresentadas na Tabela 10 , referem-se às correlações entre os escores discriminantes padronizados e cada variável independente.

Tabela 10: Cargas Discriminantes

\begin{tabular}{lc} 
& Furção 1 \\
Rect & 0,734 \\
Fpm & $-0,619$ \\
Icms & 0,225 \\
\hline
\end{tabular}

Fonte: Elaborado pelos autores.

As cargas discri-minantes indicam a importância relativa das variáveis independentes, pois refletem o respectivo poder discri-minatório dos grupos focalizados. A maior carga para Receita Tributária per capita corrobora o fato de ter sido esta a primeira a ser incluída no modelo discriminante pelo método stepwise. A correlação entre a variável transformada FPM per capita e os escores discriminantes apresenta sinal negativo, isto é, são inversamente proporcionais. O oposto ocorre com as outras variáveis.

A conclusão da análise discriminante é que as variáveis Receita Tributária per capita, FPM per capita e Quota-parte de ICMS per capita discriminam os grupos 2 e 5 que apresentam alta e baixa riqueza, respectivamente, porém são similares nos índices de longevidade e escolaridade. Os resultados indicam que a variável FPM per capita, como recurso disponível para a realização de investimentos sociais, parece não cumprir, efetivamente, o seu papel redistributivo em relação aos municípios dos dois grupos, dado que a maior carga discriminante foi para Receita Tributária per capita e a correlação entre esta variável e FPM per capita está muito distante do valor 1 , em módulo.

A variável mais importante para a discriminação é a Receita Tributária per capita, isto é, os recursos que o município pode obter por si mesmo. A variável FPM per capita está próxima da Receita Tributária em importância. A variável Quota-parte de ICMS per capita também discrimina, porém sua importância é bem menor do que a das outras duas.

Finalmente, tem-se, na Tabela 11, a matriz das classificações dos municípios a posteriori, com base no modelo discriminante, e sua comparação com as classificações $a$ priori.

Tabela 11: Resultados da Classificação

\begin{tabular}{|c|c|c|c|}
\hline & \multicolumn{2}{|c|}{ Clarsificação Previrta } & \\
\hline Classificaçä o original & gtupo 2 & gxtupo 5 & total \\
\hline$g \pm x+p 02$ & 43 & 12 & 55 \\
\hline gtupo 5 & 10 & 74 & 84 \\
\hline \multicolumn{4}{|l|}{ Em porcertigem } \\
\hline gxtupo 2 & $78,2 \%$ & $21,6 \%$ & 100,0 \\
\hline $8+7+7 p 05$ & $11,9 \%$ & $88,1 \%$ & 100,0 \\
\hline
\end{tabular}

Fonte: Elaborado pelos autores.

A magnitude da porcentagem de acertos é uma informação importante porque reflete a qualidade de ajuste do modelo de classificação gerado pela análise discriminante. A Tabela 11 revela que 43 casos do grupo 2 e 74 casos do grupo 5 foram classificados corretamente, perfazendo o total de 117 , o que corresponde a $84 \%$ dos casos analisados.

\section{CONCLUSÕES}

O presente artigo teve como objetivo indicar se os grupos de municípios paulistas, estabelecidos pelo Índice Paulista de Responsabilidade Social, mais especificamente municípios com alto índice de riqueza e baixos índices sociais e municípios desfavorecidos tanto em riqueza quanto na área social, são discriminados pelos va- 
lores per capita de transferências de FPM, quota-parte de ICMS e Receita Tributária arrecadada.

O interesse em comparar as receitas municipais surgiu da constatação da existência de diferentes níveis de riqueza entre os grupos e, por outro lado, desempenhos próximos e pobres quanto a indicadores sociais, portanto, cabendo a indagação de que as transferências governamentais, que são recursos destinados para financiar ações governamentais, sobretudo o FPM, não estariam contribuindo para tornarem mais eqüitativas as condições de investimentos dos dois grupos, haja vista que os municípios mais ricos têm maior capacidade tributária e, assim, os dois grupos poderiam estar partindo de condições financeiras diferentes.

O volume de recursos disponíveis no âmbito local para aplicação nas áreas social e econômica depende da capacidade fiscal de cada município e dos mecanismos existentes de redistribuição de recursos. A eficácia do investimento depende também da qualidade do gasto e da qualidade da gestão pública. Dada a maior capacidade dos municípios de grande porte de arrecadação de receitas próprias devido às características dos tributos municipais, espera-se que os critérios de participação dos municípios nas receitas da União e do estado sejam eficazes quanto à função redistributiva. Todavia, de acordo com a revisão teórica e com a análise dos dados empíricos realizados neste trabalho, isso nem sempre acontece. Por outro lado, não se deve esquecer que certamente existem mais problemas nos municípios maiores do que nos municípios menores e os recursos podem não ser suficientes para fazer frente às demandas sociais, o que pode explicar o baixo resultado social dos mesmos. Essa questão pode nortear estudos futuros.

As médias das três receitas públicas per capita analisadas são diferentes entre os grupos. O primeiro grupo apresenta maior valor de Receita Tributária per capita e de Quota-parte de ICMS per capita e menor valor de FPM per capita quando comparado ao outro grupo.

A análise do relacionamento entre as variáveis indica que quanto maior for a capacidade fiscal dos municípios, menor será a parcela de FPM per capita recebida, mas não de maneira proporcional. A análise discriminante sinaliza que as variáveis Receita Tributária per capita e FPM per capita são as que mais discriminam os dois grupos de municípios e o poder de discriminação de ambas é bem próximo.

Sendo assim, é possível dizer que, no caso específico dos dois grupos analisados, os critérios de distribuição do FPM ou não estão contribuindo de forma efetiva na aplicação das receitas disponíveis ou a gestão dos governos municipais não vence o desafio de assegurar condições mínimas de bem-estar social e promover o desenvolvimento econômico em âmbito local.

A análise de desempenho da gestão municipal não deve se ater à quantidade de recursos disponíveis, mas também a como tais recursos são aplicados. Os baixos índices sociais em ambos os grupos e, principalmente, no grupo com maiores recursos fiscais sugerem a existência de problemas de gestão. A distribuição dos gastos públicos entre as diversas funções de governo e os resultados alcançados comparativamente ao montante aplicado são aspectos que também precisam ser olhados com cuidado, ficando, neste trabalho, como sugestão de pesquisas futuras.

Recomenda-se a repetição da análise para outras seleções de grupos de municípios, pois, não se pode afirmar que esse fato se repete entre os outros grupos de municípios do estado de São Paulo e nem entre os municípios do Brasil; porém, sua ocorrência é um sinal concreto da necessidade de se reavaliarem os critérios de distribuição do FPM.

Outra sugestão é relativa à classificação dos municípios feita pela Fundação SEADE. Além da capacidade de geração de riqueza, seria interessante incluir como critério para agrupamento a disponibilidade de recursos para executar políticas públicas.

Em um contexto de análise mais abrangente, os autores sugerem, para estudo futuro, o impacto da criação de municípios no Brasil tanto nas finanças públicas quanto no desempenho em políticas sociais, principalmente aquelas de caráter universal, haja vista que 3000 municípios têm $90 \%$ de sua receita originária dos fundos de participação. Em relação às finanças públicas, a maior proporção de recursos transferidos pode ser sinal de indisciplina fiscal, uma vez que os políticos estão mais próximos da população e cobrar tributos pode significar redução de votos no futuro. E quanto às políticas sociais, o fato sinaliza que há uma fragmentação na disponibilização de recursos, gerando dificuldades para a criação e manutenção de estruturas necessárias ao atendimento das demandas sociais, o que pode estar sobrecarregando os municípios maiores. Contudo, essa última questão deve ser vista com cuidado, levando em consideração o desenho institucional de cada política social, com destaque para as relações intergovernamentais. Tais aspectos não foram abordados neste artigo, pois extrapolam o seu foco.

\section{REFERÊNCIAS}

ABRUCIO, Fernando Luiz; COUTO, Cláudio Gonçalves. A redefinição do papel do Estado no âmbito local. São Paulo em Perspectiva. São Paulo, v. 10, n.3, p.40-47, jul./set.1996.

AFFONSO, Rui. Os municípios e os desafios da federação no Brasil. São Paulo em perspectiva. São Paulo, v. 10, n.3, p. 3-10, jul./set. 1996.

BOVO, José Murai. Gastos sociais dos municípios e desequilíbrio financeiro. Revista de Administração Pública. Rio de Janeiro, v.35, n. 1, p. 93-117, jan./fev. 2001.

BRASIL. Constituição (1988). Constituição da República Federativa do Brasil. Coleção Saraiva de legislação. 21. ed. São 
Paulo: Saraiva, 2001.

Decreto-Lei $\mathrm{n}^{\circ} 1.881$ de 27 de agosto de 1981. Disponível em: <http://www.senado.gov.br>. Acesso em: 20 Jan. 2005.

BRASIL. Lei Complementar $\mathrm{n}^{\circ} 91$ de 22 de dezembro de 1997. Disponível em: <http://www.senado.gov.br>. Acesso em: 20 Jan. 2005.

FUNDAÇÃO SEADE. Índice Paulista de Responsabilidade Social: metodologia. Disponível em: <http://www.seade.gov.br/ produtos/iprs/pdf/metodologia.pdf $><$ Acesso em: 20 Jan. $2005 \mathrm{a}$.

FUNDAÇÃO SEADE. Índice Paulista de Responsabilidade Social: consulta. Disponível em: <http://www.al.sp.gov.br/web/ forum/iprs03/index_iprs.htm $><$ Acesso em: 20 Jan. 2005b. HAIR JR., Joseph F.; TATHAM, Ronald L.; ANDERSON, Rolph E.; BLACK, William. Análise multivariada de dados. $5^{a}$ ed. Porto Alegre: Bookman, 2005.

OSZLAK, Oscar. Estado e Sociedade: novas regras de jogo? In: FELICÍSSIMO, JR. et al. (Coord.) Sociedade e Estado: superando fronteiras. São Paulo: FUNDAP, 1998. 